\title{
Análise epidemiológica dos casos de Leishmaniose Tegumentar Americana em um
} município do Triângulo Mineiro

\author{
Epidemiological analysis of the cases American Cutaneous Leishmaniasis in a \\ municipality of Triângulo Mineiro
}

\section{Análisis epidemiológico de casos de Leishmaniasis Cutánea Americana en un municipio de Triângulo Mineiro}

Recebido: 02/07/2019

Aprovado: 16/11/2019

Publicado: 17/02/2020

\section{Hellen Cristina Bernardes ${ }^{1}$ Ana Flávia Ferreira dos Santos ${ }^{2}$ Daniel Gohm ${ }^{3}$ Lineker Fernandes Dias ${ }^{4}$ Keline Medeiros de Araújo Vilges ${ }^{5}$ Stefan Vilges de Oliveira ${ }^{6}$}

Este estudo tem o objetivo de analisar o perfil epidemiológico da Leishmaniose Tegumentar Americana no município de Patrocínio, Minas Gerais, Brasil, no período de 2007 a 2018. Trata-se de uma pesquisa epidemiológica descritiva a partir dos dados do Sistema de Informação de Agravos de Notificação do Ministério da Saúde. Nesta cidade foram notificados 21 casos no período, com uma incidência média de 0,21 casos de Leishmaniose Tegumentar Americana a cada 10 mil habitantes. 0 perfil dos indivíduos acometidos foi composto por homens $(90,48 \%)$, brancos $(71,43 \%)$, entre 50 e 64 anos de idade $(33,33 \%)$, moradores da zona urbana $(90,48 \%)$, com ensino fundamental completo $(23,81 \%)$ e com a apresentação clínica na forma cutânea $(85,71 \%)$. Todos os casos eram novos e evoluíram para cura em 80,59\%. Embora a Leishmaniose Tegumentar Americana se manifeste com uma incidência baixa no município, observa-se a manutenção da sua ocorrência ao longo dos doze anos analisados, figurando-se como uma doença endêmica no local.

Descritores: Leishmaniose; Prevenção de doenças; Vigilância em saúde pública; Zoonoses.

This study aims to analyze the epidemiological profile of American cutaneous leishmaniasis in the municipality of Patrocínio, Minas Gerais, Brazil, from 2007 to 2018. It is a descriptive epidemiological research, from data of the Information System for notifiable diseases of the Ministry of Health. In this city, 21 cases were reported in the period, with an average incidence of 0.21 cases of American cutaneous leishmaniasis for every 10,000 inhabitants. The profile of individuals affected consisted of men (90.48\%), white (71.43\%), from 50 to 64 years (33.33\%), residents of urban area (90.48\%) with complete primary education (23.81\%) and with clinical presentation of cutaneous form (85.71\%). All cases were new and evolved to cure in $80.59 \%$. Although American Cutaneous Leishmaniais manifests itself with low incidence in the municipality, it is observed maintenance of its occurrence over the twelve years analyzed, appearing as an endemic disease in the local.

Descriptors: Leishmaniasis; Disease prevention; Public health surveillance; Zoonoses.

Este estudio tiene el objetivo de analizar el perfil epidemiológico de la Leishmaniasis tegumentar Americana en el municipio de Patrocinio, Minas Gerais, Brasil, en el período de 2007 a 2018. Se trata de una investigación epidemiológica descriptiva a partir de los datos del Sistema de Información de agravios de Notificación del Ministerio de Salud. En esta ciudad se notificaron 21 casos en el período, con una incidencia media de 0,21 casos de Leishmaniosis tegumentar Americana por cada 10 mil habitantes. El perfil de los individuos afectados estaba compuesto por hombres (90,48\%), blancos (71,43\%), entre 50 y 64 años de edad (33,33\%), residentes de la zona urbana $(90,48 \%)$, con educación primaria completa $(23,81 \%)$ y con la presentación clínica en forma cutánea $(85,71$ por ciento). Todos los casos eran nuevos y evolucionaron para la curación en un 80,59\%. Aunque la Leishmaniasis tegumentar Americana se manifiesta con una incidencia baja en el municipio, se observa el mantenimiento de su ocurrencia a lo largo de los doce años analizados, considerándose como una enfermedad endémica en el local.

Descriptores: Leishmaniasis; Prevención de enfermedades; Vigilancia en salud pública; Zoonosis.

\footnotetext{
1. Bióloga. Discente do curso de Graduação em Medicina pela Universidade Federal de Uberlândia (UFU), Uberlândia/MG, Brasil. ORCID: 0000-0002-6220-3853 E-mail: hellencristinabernardes@yahoo.com.br

2. Discente do curso de Graduação em Medicina da UFU, Uberlândia/MG, Brasil. ORCID: 0000-0002-2609-2009 E-mail: anafdsantos@hotmail.com.br

3. Discente do curso de Graduação em Medicina pela UFU, Uberlândia/MG, Brasil. ORCID: 0000-0003-1960-4544 E-mail: danielgohm2009@hotmail.com

4. Discente do curso de Graduação em Medicina pela UFU, Uberlândia/MG, Brasil. ORCID: 0000-0002-6256-7139 E-mail: linekeer_dias@hotmail.com

5. Bióloga. Doutora em Medicina Tropical. Pesquisadora Autônoma, Uberlândia/MG, Brasil. ORCID: 0000-0003-3518-2169 E-mail: kelinemed@gmail.com

6. Biólogo. Doutor em Medicina Tropical. Professor Associado do curso de graduação em Medicina da UFU, Uberlândia/MG, Brasil. ORCID: 0000-0002-5493-2765

E-mail: stefanbio@yahoo.com.br
} 


\section{INTRODUÇÃO}

A

s leishmanioses são antropozoonoses que representam um grande problema de saúde pública1․ Devido ao seu amplo espectro de manifestações clínicas, são divididas em Leishmaniose Cutânea Localizada (LCL), Leishmaniose Cutâneo Mucosa (LCM), Leishmaniose Cutâneo Difusa (LCD) e Leishmaniose Disseminada (LD), todas variantes da Leishmaniose Tegumentar Americana (LTA). Além da LTA, pode ser citada também a Leishmaniose Visceral (LV)2.

No Brasil, sete espécies de Leishmania responsáveis pela LTA já foram descritas, na qual as mais importantes são: Leishmania (Leishmania) amazonensis, L. (Viannia) guyanensis e L. (V.) braziliensis. Os vetores são insetos flebotomíneos, pertencentes ao gênero Lutzomyia, popularmente conhecidos como mosquito palha, tatuquira ou birigui ${ }^{3}$.

Para o tratamento da LTA são utilizados os antimoniais pentavalentes como drogas de primeira escolha. Em casos de resistência ou de indisponibilidade é indicado o uso de medicamentos de segunda escolha, como anfotericina B e pentamidina ${ }^{4}$. 0 alto custo, as dificuldades de administração, o longo tempo de tratamento, a toxicidade e o aumento da morbidade são fatores que limitam o uso dos medicamentos, fato que contribui para a não adesão terapêutica e predisposição de complicações como a extensão da doença nas membranas mucosas ou lesões desfigurantes ${ }^{1}$.

A LTA é considerada uma das afecções dermatológicas que merece atenção, devido à sua magnitude, levando em consideração a ocorrência de deformidades no ser humano, específicas dessas doenças e também o envolvimento psicológico, com reflexos no campo social e econômico ${ }^{3}$. As leishmanioses estão entre as doenças negligenciadas devido aos recursos limitados investidos em seu diagnóstico, tratamento e controle, juntamente com sua forte associação à pobreza e aos conflitos sociais ${ }^{1}$.

Apresenta ampla distribuição mundial e no continente americano há registro de casos desde o sul dos Estados Unidos ao norte da Argentina, com exceção do Chile e Uruguai ${ }^{1}$. Em 2017 foram registrados 17.528 casos novos no Brasil, com coeficiente de detecção de 8,4 casos $/ 100.000$ habitantes $^{5}$. A região Norte apresenta o maior coeficiente $(43,7$ casos $/ 100.000$ habitantes), seguida das regiões Centro-Oeste (15,2 casos/100.000 habitantes), Nordeste $(7,4$ casos/100.000 habitantes), Sudeste (1,8 casos/100.000 habitantes) e Sul (0,6 casos/100.000 habitantes) ${ }^{5}$.

Diante disso, nos últimos anos, o Ministério da Saúde do Brasil tem investido na busca de novos conhecimentos e alternativas para o controle dessa endemia ${ }^{5}$. As principais linhas de pesquisa enfocam na implementação de diagnóstico laboratorial humano, tratamento de pacientes com leishmaniose, avaliação da eficácia das estratégias de controle vetorial e de reservatório e novas tecnologias que podem contribuir para a implementação de medidas de vigilância e controle das leishmanioses no país ${ }^{6}$.

Em razão disso, a leishmaniose é uma doença que deve ser notificada e investigada pelos serviços de saúde, por meio da ficha de notificação e investigação padronizada pelo Sistema de Informação de Agravos de Notificação ${ }^{7}$. 0 seu registro nos sistemas oficiais de vigilância em saúde é importante para o conhecimento do seu perfil epidemiológico e o acompanhamento com vistas a propor estratégias de prevenção e controle ${ }^{1}$.

Por ser um agravo de importância clínica e epidemiológica no Brasil, com diversos fatores geográficos, de vigilância em saúde e ecológicos envolvidos na dinâmica da doença ${ }^{8}$, justifica-se analisar o perfil epidemiológico da LTA no município de Patrocínio, Minas GeraisMG. Diante disso, este estudo tem como objetivo analisar o perfil epidemiológico da LTA no município de Patrocínio, Minas Gerais, Brasil, no período de 2007 a 2018. 


\section{MÉTODO}

Este é um estudo epidemiológico, de caráter descritivo, baseado em dados secundários da LTA, notificados na cidade de Patrocínio - MG, no período de 1 de janeiro de 2007 a 31 de dezembro de 2018. Esses dados são oriundos do Sistema de Informação de Agravos de Notificação - SINAN e foram disponibilizados de acordo com o protocolo 25820006595201811, que autoriza o acesso a base dos dados epidemiológicos, disponibilizada pelo Ministério da Saúde (MS) do Brasil pelo Sistema de Informação ao Cidadão.

O município de Patrocínio está localizado no Triangulo Mineiro, na Mesorregião Alto Paranaíba há cerca de 419,9 km de Belo Horizonte e tem uma área territorial de 2.874,344 Km² 9. Possui 82.471 habitantes, sendo que 72.758 têm residência na cidade e, 9.713, tem residência na zona rural $^{9}$. Considerando a distribuição espacial da população, a densidade demográfica é de 28,69 hab $/ \mathrm{km}^{2}{ }^{10}$. A base da economia é a agricultura e a pecuária, representados pela cafeicultura e gado leiteiro, responsáveis pela maior parte da arrecadação de ICMS do município e segundo informações do Atlas do Desenvolvimento Humano no Brasil 2013, o município está situado na faixa de Desenvolvimento Humano Alto (IDHM entre 0,700 e $0,799)^{10}$.

Os critérios de inclusão aplicados neste estudo foram todos os casos notificados de LTA no SINAN, que informaram residir no município de Patrocínio e que foram notificados entre os anos de 2007 a 2018.

Foram avaliadas as variáveis em relação ao perfil demográfico: casos confirmados em pessoas residentes no município de Patrocínio - MG; por ano; por mês da notificação, sexo (masculino e feminino); faixa etária em anos categorizados $(1$ a $4 ; 15$ a $19 ; 20$ a $34 ; 35$ a $49 ; 50$ a 64; 65 a 79); zona de residência (urbana; rural e periurbana; ignorado ou em branco); escolaridade (analfabeto, $1^{\underline{a}}$ a $4^{\underline{a}}$ série incompletas, $4^{\underline{a}}$ série completa, $5^{\underline{a}}$ a $8^{\underline{a}}$ incompleta, ensino fundamental completo, ensino médio incompleto/completo, educação superior incompleta/completa, ignorado) e raça/cor (branca, preta, amarela, parda, indígena ou ignorado).

Em relação ao perfil clínico, laboratorial e de tratamento, foram avaliadas as seguintes variáveis: forma clínica (cutânea e mucosa); droga inicial de tratamento (Antimonial Pentavalente; Anfotericina b; Anfotericina b lipossomal e outras drogas); dose prescrita $(<10$; $>$ ou $=10$ e $<20 ; 15 ;>15$ e $<20$; $>$ ou $=20$ e ignorado ou em branco) e o tipo de entrada (caso novo; recidiva e transferência) e evolução do caso (cura; abandono; transferência e ignorados ou em branco).

As informações obtidas pela base de dados do SINAN foram acessadas em 30 de abril de 2019 e foram transformadas em tabelas e, subsequentemente, foi utilizado o programa Microsoft Excel, versão 2013, para construção dos gráficos. Foi realizada análise estatística descritiva e os dados foram apresentados por números brutos, frequência relativa, percentual e média dispostos em tabelas e gráficos.

Para o cálculo da incidência anual utilizou-se o número de casos de LTA notificados por anos / pela população do município de Patrocínio X 10 mil habitantes. A informação sobre a população anual foi obtida pelas estimativas de população fornecidas pelo IBGE e disponibilizadas pelo Departamento de Informática do Sistema Único de Saúde do Brasil (DATASUS) ${ }^{11}$.

Este estudo dispensa a apreciação pelo Comitê de Ética em Pesquisa, visto que utiliza de dados secundários não possibilitando a identificação nominal dos sujeitos da pesquisa.

\section{RESULTADOS}

Foram notificados 21 casos de LTA na cidade de Patrocínio - MG no intervalo de 2007 a 2018. Os anos de 2010 e 2015 foram os que apresentaram o maior número de casos (4 casos respectivamente), correspondendo a 38,1\% dos casos. Nos anos de 2008, 2017 e 2018 não 
foram registrados casos de LTA. A incidência média foi de 0,21 casos a cada 10 mil habitantes (Figura 1).



Figura 1. Casos de Leishmaniose Tegumentar Americana, segundo os dados do Sistema de Informação de Agravos de Notificação. Patrocínio, Minas Gerais, 2007 a 2018.

Fonte: Sistema de Informação de Agravos de Notificação - SINAN.

Os casos são distribuídos em todos os meses do ano (exceção de agosto e outubro) com maior frequência de registro nos meses de maio e julho $(38,1 \%)$ (Figura 2). Observou-se que os indivíduos mais acometidos pela doença são do sexo masculino (90,48\% dos casos), principalmente nas faixas etárias de 50 a 64 anos (33,33\%) (Figura 3).

Quanto à zona de residência, pode-se observar que a doença acometeu, predominantemente, indivíduos que moravam em zona urbana, com 90,48\% das ocorrências. 0 nível de escolaridade dos indivíduos mais acometido foi o ensino fundamental completo $23,81 \%$ e a LTA acometeu em sua maioria pessoas brancas, correspondendo a $71,43 \%$ nestas (Tabela 1).

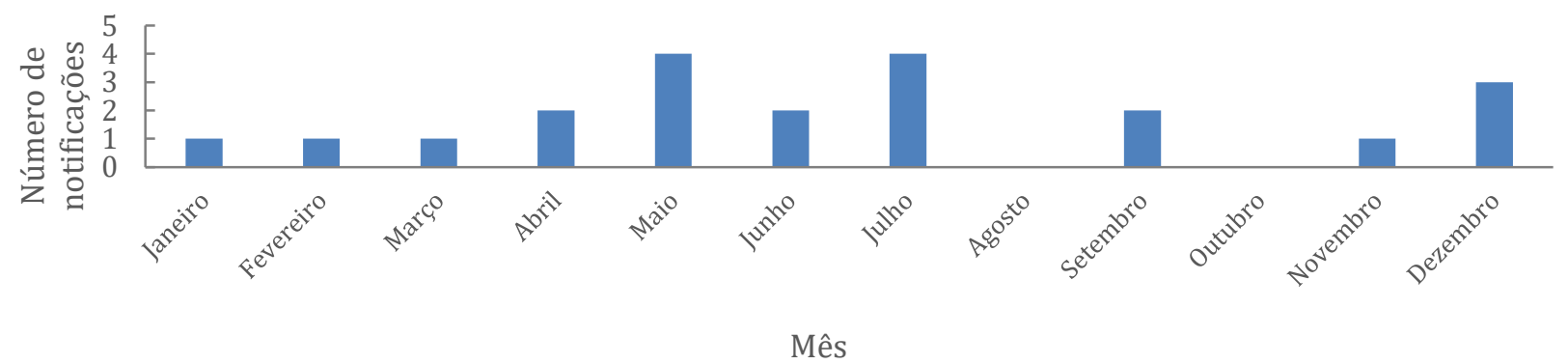

Figura 2. Caso de Leishmaniose Tegumentar Americana de acordo com o mês de ocorrência, segundo dados do Sistema de Informação de Agravos de Notificação. Patrocínio, Minas Gerais, 2007-2018.

Fonte: Sistema de Informação de Agravos de Notificação - SINAN. 


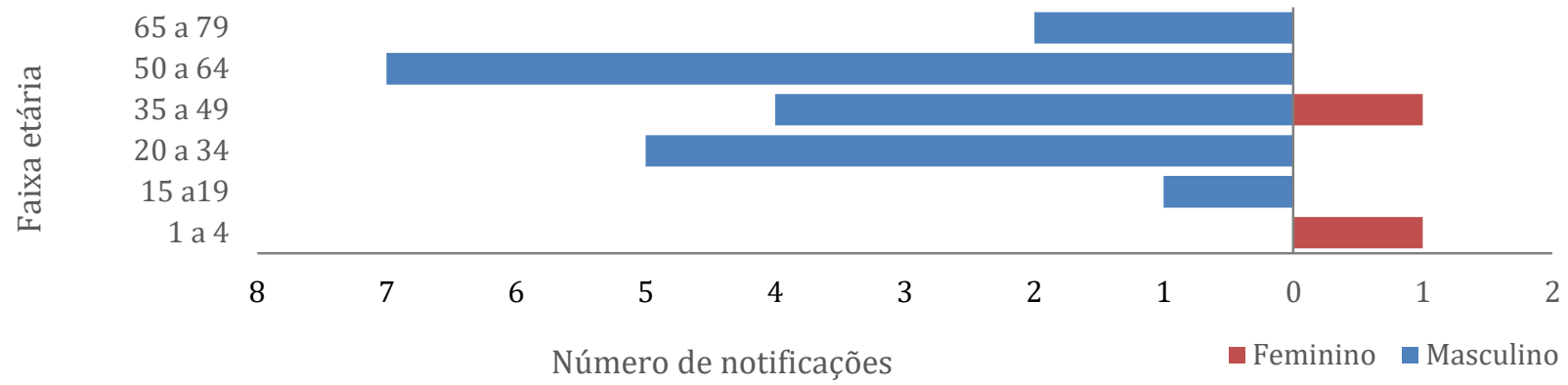

Figura 3. Ocorrência de Leishmaniose Tegumentar Americana conforme faixa etária e sexo segundo dados do Sistema de Informação de Agravos de Notificação. Patrocínio, Minas Gerais, 2007- 2018.

Fonte: Sistema de Informação de Agravos de Notificação - SINAN.

Tabela 1. Perfil sociodemográfico em casos notificados da Leishmaniose Tegumentar Americana, segundo dados do Sistema de Informação de Agravos de Notificação. Patrocínio, Minas Gerais, 2007- 2018.

\begin{tabular}{|c|c|c|}
\hline Variáveis & Frequência & Percentual \\
\hline \multicolumn{3}{|l|}{ Zona de residência } \\
\hline Urbana & 19 & 90,48 \\
\hline Rural & 2 & 9,52 \\
\hline Periurbana & 0 & 0,0 \\
\hline Ignorado ou em branco & 0 & 0,0 \\
\hline \multicolumn{3}{|l|}{ Escolaridade } \\
\hline Analfabeto & 1 & 4,76 \\
\hline 1ㅁ a 4⿳⺈ série incompleta do ensino fundamental & 3 & 14,29 \\
\hline $5^{\mathrm{a}}$ a $8^{\mathrm{a}}$ série incompleta do ensino fundamental & 2 & 9,52 \\
\hline Ensino fundamental completo & 5 & 23,81 \\
\hline Ensino médio completo & 3 & 14,29 \\
\hline Educação superior incompleta & 1 & 4,76 \\
\hline Ignorado ou em branco & 6 & 28,57 \\
\hline \multicolumn{3}{|l|}{ Raça/Cor } \\
\hline Branca & 15 & 71,43 \\
\hline Preta & 1 & 4,76 \\
\hline Amarela & 0 & 0,0 \\
\hline Parda & 5 & 23,81 \\
\hline Indígena & 0 & 0,0 \\
\hline Total & 21 & 100,0 \\
\hline
\end{tabular}

A forma clínica de maior frequência foi a cutânea, compreendendo 85,71\% dos casos. Os indivíduos foram tratados, exclusivamente, com antimonial pentavalente com uma dose prescrita variando entre $>15$ e $<20 \mathrm{mg} / \mathrm{kg} / \mathrm{dia}$ em 33,33\%. Todos os casos da presente série eram novos e evoluíram para cura em $80,59 \%$ (Tabela 2 ).

Tabela 2. Perfil clínico, laboratorial e de tratamento dos casos notificados da Leishmaniose Tegumentar Americana, segundo os dados do Sistema de Informação de Agravos de Notificação Patrocínio, Minas Gerais, 2007- 2018.

\begin{tabular}{lcc}
\hline Variáveis & Frequência & Porcentagem \\
\hline Forma clínica & & \\
\hline Cutânea & 18 & 85,71 \\
\hline Mucosa & 3 & 14,29 \\
\hline Droga inicial & & \\
\hline Antimonial pentavalente & 21 & 100,0 \\
\hline
\end{tabular}




\begin{tabular}{lcc}
\hline Anfotericina b & 0 & 0,0 \\
\hline Anfotericina b lipossomal & 0 & 0,0 \\
\hline Outras drogas & 0 & 0,0 \\
\hline Dose prescrita mg/kg/dia & & \\
\hline$<10$ & 3 & 14,29 \\
\hline$>$ ou $=10$ e $<20$ & 6 & 28,57 \\
\hline 15 & 1 & 3,76 \\
\hline$>15$ e $<20$ & 7 & 9,53 \\
\hline$>$ ou = 20 & 2 & 9,52 \\
\hline Ignorado ou em branco & 2 & \\
\hline Tipo de entrada & & 0,0 \\
\hline Caso novo & 21 & 0,0 \\
\hline Recidiva & 0 & \\
\hline Transferência & 0 & 80,95 \\
\hline Evolução do caso & & 9,52 \\
\hline Cura & 17 & 9,52 \\
\hline Transferência & 2 & 100,0 \\
\hline Ignorado ou em branco & 2 & 21 \\
\hline Total & & \\
\hline Fon & & \\
\hline
\end{tabular}

Fonte: Sistema de Informação de Agravos de Notificação - SINAN.

\section{DISCUSSÃo}

Observa-se que o município de Patrocínio - MG possui uma incidência baixa de LTA, em relação a sua população, que é de, aproximadamente, 85 mil habitantes. Pode-se perceber que o perfil epidemiológico do agravo inclui, majoritariamente, homens, da zona urbana, de 50 a 64 anos, brancos e acometidos pela forma cutânea.

No Brasil, nos anos de 2007 a 2017, foram notificados 216.526 casos, sendo, destes, $72,7 \%$ homens, $63,1 \%$ na faixa etária entre 20 e 59 anos, $60,7 \%$ pardos, $53,9 \%$ procedentes da zona rural e 93,8\% apresentando a forma cutânea ${ }^{12}$.

Já na região Sudeste, o número de notificações foi 19.205, aproximadamente $8,8 \%$ do total no país. Compôs-se o perfil epidemiológico, em sua maioria, por homens $(62,85 \%)$, de 20 a 59 anos $(59 \%)$, brancos $(40,2 \%)$, procedentes da zona urbana $(51,3 \%)$ e com manifestações cutâneas $(88 \%)^{12}$.

0 estado de Minas Gerais, por sua vez, englobou cerca de $70 \%$ dos casos da região sudeste (13.437 notificações), acometendo homens (61,8\%), entre 20 e 59 anos de idade $(59,4 \%)$, pardos $(43,5 \%)$, procedentes da zona rural $(48,3 \%)$ e apresentando a forma cutânea $(91,6 \%)^{13}$.

No município de Patrocínio, os fatores sexo, faixa etária e forma de apresentação do agravo, são equivalentes nos âmbitos nacional, regional, estadual e municipal ${ }^{1}$. Entretanto, o país e o estado de Minas Gerais apontaram pardos e a zona rural como mais prevalentes ${ }^{12,13}$, em contrapartida ao encontrado no Sudeste do país e no município de Patrocínio, nos quais há mais brancos procedentes da zona urbana, nas notificações da doença ${ }^{12,13}$.

Estudos mostram que os topos das colinas, áreas planas ou quase planas, quando recobertas por vegetação arbustiva, podem constituir-se em locais propícios para a convivência de animais reservatórios, vetores e agentes etiológicos ${ }^{14}$. Quando há expansão geográfica associada à modificação de ambientes florestais primários pela atuação humana, antropização dos ciclos evolutivos da Leishmania com o desmatamento, abertura de estradas e urbanização de áreas primitivas, a tendência dos insetos vetores é migrar para áreas onde o solo não é muito acidentado e onde há pequenas extensões de matas residuais próximas do ambiente alterado ${ }^{15}$.

Os flebotomíneos alados são insetos que, em geral, possuem pouca tendência a se afastarem dos seus abrigos naturais. Porém, em casos excepcionais, o flebotomíneo pode se dispersar até 1.500 metros, mas, usualmente, o inseto não ultrapassa de 200 a $500 \mathrm{~m}^{16}$.

0 relevo de Patrocínio se classifica em $60 \%$ plano, $30 \%$ ondulado e $10 \%$ montanhoso, coberto por vegetação do tipo cerrado com predomínio de campos sujos, campos limpos, matas 
ciliares (galerias) e com raros nichos de campos rupestres 9.0 entorno da cidade de Patrocínio é circundado, majoritariamente, por relevo plano e ondulado, vegetação arbustiva e há grande interferência humana na natureza, devido ao desenvolvimento da pecuária e agricultura ${ }^{9}$. Com isso, animais reservatórios de muitas doenças infectocontagiosas, usualmente, deslocam-se para matas residuais inseridas no limite urbano e periurbano ${ }^{14}$.

Logo, é possível depreender que a incidência de casos de LTA na cidade de Patrocínio MG tem prevalência urbana, devido às características do relevo e vegetação da cidade que favorecem a manutenção de agentes etiológicos da Leishmania sp. Ademais, a intensa atividade agropecuária, que favorece a translocação de animais reservatórios e vetores da leishmaniose para áreas verdes presentes no perímetro urbano, expõe a população, que reside próximo a essas áreas a um maior risco de contato com o protozoário.

Quanto à raça, é importante que esses dados sejam interpretados a partir dos dados demográficos da população residente por cor ${ }^{17}$. A leishmaniose não tem predileção por raça, mas sua incidência é proporcional à etnia mais prevalente de um determinado município ${ }^{17}$. Dessa forma, justifica-se a maior prevalência de leishmaniose na raça branca $(71,43 \%)$, seguida da raça parda $(23,81 \%)$ e negros $(4,76 \%)$ em Patrocínio devido a população ser, em sua maioria, da raça branca $(59,2 \%)$, seguida da raça parda (32\%), negros $(7,8 \%)$, amarelos $(0,8 \%)$ e indígenas $(0,07 \%)$. Não foram verificados eventos nas raças amarelos e indígenas ${ }^{18}$.

A prevalência da LTA em homens com idade entre 50 a 64 anos (90,48\% dos casos) foi significativa com relação ao sexo feminino ${ }^{13}$. Segundo estudo que avaliou os aspectos epidemiológicos da LTA no Estado de Alagoas ${ }^{19}$, os homens seriam os mais acometidos pela maior exposição aos fatores de risco para o surgimento da doença. A maior incidência de casos no sexo masculino vem sendo relacionada ao tipo de atividade ocupacional dos homens, predominantemente atividades rurais, como agricultura, pecuária e garimpo ${ }^{20}$.

Nesta pesquisa, constatou-se que a faixa etária predominante foi a de 50 a 64 anos. Esses resultados são diferentes dos dados de LTA em, Manaus, $\mathrm{AM}^{21}$, na qual a faixa etária de maior prevalência foi a de 20 anos. Segundo o MS ${ }^{1}$, isso se justifica pelo fato dos indivíduos nessa faixa etária estarem na fase produtiva com encontro ocupacional nas atividades laborais, as quais se relacionam muitas vezes ao desmatamento (entradas em áreas florestais) e atividades militares e ao trabalho doméstico e/ou na lavoura (subsistência), o que demonstra o maior contato com os vetores transmissores que causam essa patologia. No município de Patrocínio os resultados são divergentes devido às atividades trabalhistas estarem relacionadas, principalmente, à homens com idade de 30 a menos de $60 \operatorname{anos}^{22}$.

Quando comparado a cidades do mesmo estado, é possível salientar a importância do agravo na cidade. Alfenas, por exemplo, possui cerca de 75 mil habitantes ${ }^{23}$ e apenas 3 casos de LTA notificados no período de 2007 a 201713. Ituiutaba, por sua vez, possui uma população estimada de cerca de 100 mil pessoas ${ }^{23}$, entretanto, também, possui 3 casos notificados no SINAN no mesmo período ${ }^{13}$. Já em Lavras, município a sudoeste do estado, com estimativa populacional de 102 mil habitantes ${ }^{23}$, notificou 10 casos no período ${ }^{13}$. Enquanto Viçosa, com aproximadamente 78 mil moradores ${ }^{23}$, apresentou 17 pessoas identificadas com a enfermidade durante o período pesquisado ${ }^{13}$. No entanto, Paracatu, município a $253 \mathrm{~km}$ de distância de Patrocínio, com aproximadamente a mesma faixa etária populacional ${ }^{23}$ foram notificados 145 casos do agravo ${ }^{13}$.

Já em comparação com cidades de todo o país, nota-se uma discrepância entre os diversos municípios. Em Senador Canedo, município do estado de Goiás com mais de 100 mil habitantes ${ }^{23}$, possui apenas 5 casos notificados no período da pesquisa ${ }^{24}$. Algumas cidades não apresentaram nenhum registro do agravo entre 2007 e 2017, tais como São Pedro da Aldeia no Rio de Janeiro ${ }^{25}$ (cerca de 100 mil habitantes $^{23}$ ) e Santana do Livramento no Rio Grande do Sul ${ }^{26}$ (aproximadamente 80 mil moradores) ${ }^{23}$. Por outro lado, alguns municípios notificaram um alto índice das infecções pelo protozoário, como Cruzeiro do Sul no estado do Acre com mais de 600 
casos registrado no SINAN ${ }^{27}$, sendo que o local possui uma população menor que 90 mil habitantes ${ }^{23}$.

Um possível fator de limitação das análises é o grande número de subnotificações do agravo em algumas localidades ${ }^{28}$. Além disso, é importante salientar que muitos casos são transferidos para os centros de saúde de referência da região ${ }^{1}$, como a cidade de Uberlândia, local onde são diagnosticados casos da doença advindos de Patrocínio.

Apesar da divergência de dados ao redor do país, ressalta-se a necessidade de uma melhora no diagnóstico dos casos suspeitos, possibilitando reduzir a subnotificação do agravo ${ }^{1}$. Ademais, Patrocínio conta com um grande contingente de cães abandonados nas ruas, os quais podem ser reservatórios do protozoário e não são diagnosticados e tratados da forma adequada, além de os casos positivados não serem divulgados à população, o que possibilita uma menor valorização desse problema de saúde Pública1.

Na forma clínica 85,71\% dos casos foram caracterizados pela forma cutânea da doença. Estudos similares que buscaram avaliar a incidência da doença no Brasil e em Montes Claros, cidade de Minas Gerais, verificaram que a forma cutânea foi também, a mais prevalente ${ }^{15,28}$. Nesse sentido, verifica-se que a forma da doença em Patrocínio seguiu o mesmo padrão relatado em outros estudos de nível nacional e, também, em outra cidade do mesmo estado.

Os indivíduos foram tratados, exclusivamente, com antimonial pentavalente, este, com uma dose prescrita variando entre $>15$ e $<20 \mathrm{mg} / \mathrm{kg} /$ dia em 33,33\% dos casos. Todos os casos da presente série eram novos e evoluíram para cura em 80,59\%. Recente trabalho de revisão apontou que o antimonial pentavalente é a droga de escolha mais utilizada para tratamento da leishmaniose e que, o parasita não apresentou sinais de resistência à droga ${ }^{29}$. Nesse sentido, depreende-se que as taxas de cura da doença, constatadas, também caminharam em consonância com as taxas de cura relatadas em outras localidades.

É importante ressaltar que além de medidas de controle da doença é necessário esclarecer a população sobre os métodos de precaução, transmissão e tratamento por agentes de saúde e população em geral ${ }^{17}$.

As atividades de educação em saúde devem estar inseridas em todos os serviços que desenvolvam as ações de vigilância e controle da LTA, requerendo o envolvimento efetivo das equipes multiprofissionais e multi-institucionais, com vistas ao trabalho articulado nas diferentes unidades de prestação de serviços ${ }^{1}$.

\section{CONCLUSÃO}

O perfil epidemiológico da LTA em Patrocínio é de maior prevalência urbana, se justificando pelas características do relevo e vegetação do município, os quais favorecem a manutenção dos seus agentes etiológicos. Ademais, a intensa atividade agropecuária da região, também, favorece o deslocamento de vetores da leishmaniose para áreas verdes presentes no perímetro urbano, expondo a população residente próxima a esses espaços a um maior risco de contato com o protozoário.

Ainda de acordo com o perfil epidemiológico, foi possível concluir que a raça mais acometida por casos de LTA foi a branca, devido a cidade possuir uma população composta, majoritariamente, por pessoas brancas. Concomitante a isso, foi observado um significativo número de casos em homens com idade de 30 a 60 anos, fator que se relaciona à idade economicamente ativa das pessoas residentes no município de Patrocínio.

A forma cutânea da LTA foi a mais encontrada, seguindo o mesmo padrão relatado em estudos científicos de nível nacional e, também, em outras cidades do mesmo estado, sendo a droga mais utilizada para o tratamento o antimonial pentavalente.

Dentre as limitações deste estudo, convém pontuar a subnotificação de casos da LTA que podem ser realidade no município analisado. Ademais, prospecta-se a necessidade de futuros trabalhos de pesquisa que busquem avaliar o correto preenchimento da ficha de notificação compulsória por profissionais de saúde atuantes na cidade. 


\section{REFERÊNCIAS}

1. Ministério da Saúde (Brasil). Secretaria de Vigilância em Saúde. Departamento de Vigilância das Doenças Transmissíveis. Manual de vigilância da leishmaniose tegumentar [Internet]. Brasília, DF: Ministério da Saúde; 2017. [citado em 30 maio 2019]. Disponível em: http://bvsms.saude.gov.br/bvs/publicacoes/manual_vigilancia_leishmaniose_tegumentar.pdf 2. Vieira ÉLM, Keesen TSL, Machado PR, Guimarães LH, Carvalho EM, Dutra WO, et al. Immunoregulatory profile of monocytes from cutaneous leishmaniasis patients and association with lesion size. Parasite Immunol. [Internet]. 2013 [citado em 30 maio 2019]; 35(2):65-72. Disponível em: https://onlinelibrary.wiley.com/doi/full/10.1111/pim.12012. DOI: https://doi.org/10.1111/pim.12012

3. Gontijo B, Carvalho MLR. Leishmaniose tegumentar americana. Rev Soc Bras Med Trop. [Internet]. 2003 [citado em 30 maio 2019]; 36(1):71-80. Disponível em: http://www.scielo.br/pdf/rsbmt/v36n1/15310.pdf. DOI: http://dx.doi: 10.1590/S003786822003000100011

4. Lima EB, Porto C, Motta JOC, Sampaio RNR. Tratamento da leishmaniose tegumentar americana. An Bras Dermatol. [Internet]. 2007 [citado em 30 maio 2019]; 82(2):111-24. Disponível em: http://www.scielo.br/pdf/abd/v82n2/a02v82n2.pdf

5. Ministério da Saúde (Brasil). Secretaria de Vigilância a Saúde. Leishmaniose Tegumentar (LT): o que é, causas, sintomas, tratamento, diagnóstico e prevenção [Internet]. 2019 [citado em 30 maio de 2019]. Disponível em: http://www.saude.gov.br/saude-de-a-z/leishmaniosetegumentar

6. Moreira RCR, Rebêlo JMM, Gama MEA, Costa JML. Nível de conhecimentos sobre leishmaniose tegumentar americana (LTA) e uso de terapias alternativas por populações de uma área endêmica da Amazônia do Maranhão, Brasil. Cad Saude Publica. [Internet]. 2002 [citado em 01 junho 2019]; 18(1):187-95. Disponível em: https://www.scielosp.org/pdf/csp/2002.v18n1/187-195/pt. DOI: http://dx.doi.org/10.1590/S0102-311X2002000100019

7. Ministério da Saúde (Brasil). SINAN Sistema de Informação de Agravos de Notificação: o SINAN [Internet]. [Brasília, DF: Ministério da Saúde]; 2016 [citado em 1 junho 2019]. Disponível em: http://portalsinan.saude.gov.br/o-sinan

8. Temponi AOD, Brito MG, Ferraz ML, Diniz SA, Silva MX, Cunha TN. Ocorrência de casos de leishmaniose tegumentar americana: uma análise multivariada dos circuitos espaciais de produção, Minas Gerais, Brasil, 2007 a 2011. Cad Saude Pública [Internet]. 2018 [citado em 01 jun 2019]; 34(2):2-14. Disponível em: http://www.scielo.br/pdf/csp/v34n2/1678-4464-csp34-02-e00165716.pdf. DOI: http://dx.doi: 10.1590/0102-311X00165716

9. Prefeitura de Patrocínio. Informações sobre o município [Internet]. Patrocínio, MG: Prefeitura; 2019 [citado em 03 jun 2019]. Disponível em: https://portal.patrocinio.mg.gov.br/pm/index.php/municipio/informacoes-sobre-omunicipio

10. Instituto Brasileiro de Geografia e Estatística. Panorama da cidade de Patrocínio-Minas Gerais [Internet]. Rio de Janeiro: IBGE; 2019 [citado em 01 jun 2019]. Disponível em: https://cidades.ibge.gov.br/brasil/mg/patrocinio/panorama

11. Departamento de Informática do SUS (Brasil). DATASUS: histórico e apresentação [Internet]. Brasília, DF: DATASUS; 2019 [citado em 01 jun 2019]. Disponível em: http://datasus.saude.gov.br/datasus

12. Departamento de Informática do SUS (Brasil). Leishmaniose Tegumentar Americana: casos confirmados notificados no Sistema de Informação de Agravos de Notificação - Brasil [Internet]. Brasília, DF: DATASUS; 2019 [citado em 03 jun 2019]. Disponível em: http://tabnet.datasus.gov.br/cgi/tabcgi.exe?sinannet/cnv/ltabr.def

13. Departamento de Informática do SUS (Brasil). Leishmaniose Tegumentar Americana: casos confirmados notificados no Sistema de Informação de Agravos de Notificação - Minas Gerais 
[Internet]. Brasília, DF: DATASUS; 2019 [citado em 03 jun 2019]. Disponível em: http://tabnet.datasus.gov.br/cgi/tabcgi.exe?sinannet/cnv/ltamg.def

14. Miranda C, Marques CCA, Massa JL. Sensoriamento remoto orbital como recurso para análise da ocorrência da leishmaniose tegumentar americana em localidade urbana da região Sudeste do Brasil. Rev Saúde Pública [Internet]. 1998 [citado em 03 jun 2019]; 32(5):455-63. Disponível em: http://observatorio.faculdadeguanambi.edu.br/wpcontent/uploads/2015/04/MIRANDA.C.-et-al-ARTIGO.pdf.

DOI: http://dx.doi.org/10.1590/S0034-89101998000500008

15. Negrão GN, Ferreira MEMC. Considerações sobre a leishmaniose tegumentar americana e sua expansão no território brasileiro. Rev Percurso [Internet]. 2014 [citado em 03 jun 2019]; 6(1):147-68.

Disponível

http://periodicos.uem.br/ojs/index.php/Percurso/article/view/49452.

D0I: http://dx.doi.org/10.4025/revpercurso.v6i1.21375

16. Miranda C, Massa JL, Marques CCA. Análise da ocorrência de leishmaniose tegumentar americana através da imagem obtida por sensoriamento remoto orbital em localidade urbana da região Sudeste do Brasil. Rev Saúde Pública [Internet]. 1996 [citado em 03 jun 2019]; 30(5):433-7.

Disponível

em:

https://www.scielosp.org/article/ssm/content/raw/?resource_ssm_path=/media/assets/rsp /v30n5/5095.pdf. DOI: http://dx.doi.org/10.1590/S0034-89101996000500005

17. Campos SS, Campos FS, Gois GC, Silva TS. Perfil epidemiológico dos pacientes com leishmaniose tegumentar americana no município de Ilhéus - Bahia. Semina Cienc Biol Saúde [Internet]. 2017 [citado em 03 jun 2019]; 38(2):155-64. Disponível em: www.uel.br/revistas/uel/index.php/seminabio/article/download/28416/23161. DOI: http://dx.doi: 10.1590/S0037-86822003000100011

18. Instituto Brasileiro de Geografia e Estatística. Censo: amostra: características da população [Internet]. Rio de Janeiro: IBGE; 2019 [citado em 01 jun 2019]. Disponível em: https://cidades.ibge.gov.br/brasil/mg/patrocinio/pesquisa/23/25888?detalhes=true

19. Rocha TJM, Barbosa ACA, Santana EPC, Calheiros CML. Aspectos epidemiológicos de leishmaniose tegumentar americana no Estado de Alagoas, Brasil. Rev Pan Amaz Saúde [Internet]. 2015 [citado em 3 jun 2019]; 6(4):49-54. Disponível em: http://scielo.iec.gov.br/pdf/rpas/v6n4/v6n4a07.pdf. DOI: http://dx.doi.org/10.5123/S217662232015000400007

20. França EL, Mandadori MN, França JL, Botelho ACF, Ferrari CKB, França ACH. Aspectos epidemiológicos da leishmaniose tegumentar americana no município de Juína, Mato Grosso, Brasil. Sci Med. [Internet]. 2009 [citado em 03 jun 2019]; 19(3):103-7. Disponível em: http://revistaseletronicas.pucrs.br/ojs/index.php/scientiamedica/article/viewFile/5231/42 62

21. Guerra JAO, Barbosa MGV, Oureiro ACSP, Coelho CP, Rosa GG, Coelho LIACR. Leishmaniose tegumentar americana em crianças: aspectos epidemiológicos de casos atendidos em Manaus, Amazonas, Brasil. Cad Saúde Pública [Internet]. 2007 [citado em 04 jun 2019]; 23(9):2215-23. Disponível em: http://www.scielo.br/scielo.php?script=sci_arttext\&pid=S0102311X2007000900029. DOI: 10.1590/S0102-311X2007000900029

22. Instituto Brasileiro de Geografia e Estatística. Censo agropecuário [Internet]. Rio de Janeiro: IBGE; 2019 [citado em 04 jun 2019]. Disponível em: https://cidades.ibge.gov.br/brasil/mg/patrocinio/pesquisa/24/76693

23. Instituto Brasileiro de Geografia e Estatística. Cidades [Internet]. Rio de Janeiro: IBGE; 2019 [citado em 15 jul 2019]. Disponível em: https://cidades.ibge.gov.br/

24. Departamento de Informática do SUS (Brasil). Leishmaniose Tegumentar Americana - casos confirmados notificados no Sistema de Informação de Agravos de Notificação - Goiás [Internet]. Brasília, DF: DATASUS; 2019 [citado em 04 jun 2019]. Disponível em: http://tabnet.datasus.gov.br/cgi/tabcgi.exe?sinannet/cnv/ltago.def 
25. Departamento de Informática do SUS (Brasil). Leishmaniose Tegumentar Americana - casos confirmados notificados no Sistema de Informação de Agravos de Notificação - Rio de Janeiro [Internet]. Brasília, DF: DATASUS; 2019 [citado em 07 jun 2019]. Disponível em: http://tabnet.datasus.gov.br/cgi/deftohtm.exe?sinannet/cnv/ltarj.def

26. Departamento de Informática do SUS (Brasil). Leishmaniose Tegumentar Americana - casos confirmados notificados no Sistema de Informação de Agravos de Notificação - Rio Grande do Sul [Internet]. Brasília, DF: DATASUS; 2019 [citado em 07 jun 2019]. Disponível em: http://tabnet.datasus.gov.br/cgi/deftohtm.exe?sinannet/cnv/ltars.def

27. Departamento de Informática do SUS (Brasil). Leishmaniose Tegumentar Americana - casos confirmados notificados no Sistema de Informação de Agravos de Notificação - Acre. [Internet]. Brasília, DF: DATASUS; 2019 [citado em 07 jun 2019]. Disponível em: http://tabnet.datasus.gov.br/cgi/deftohtm.exe?sinannet/cnv/ltaac.def

28. Xavier KD, Mendes FCF, Rossi-Barbosa, LAR. Leishmaniose tegumentar americana: estudo clínico-epidemiológico. Rev Univ Vale Rio Verde [Internet]. 2016 [citado em 07 jun 2019]; 14(2):1210-22. Disponível

em:

http://periodicos.unincor.br/index.php/revistaunincor/article/view/2880/pdf_609

29. Conti RV, Pinto Junior VL. Abordagem terapêutica da leishmaniose visceral no Brasil: revisão para clínicos. Rev Med Saúde Brasília [Internet]. 2015 [citado em 08 jun 2019]; 4(2):240-9. Disponível em: https://bdtd.ucb.br/index.php/rmsbr/article/view/6170/3966

\section{CONTRIBUIÇÕES}

Todos os autores tiveram iguais contribuições na elaboração deste artigo.

\section{Como citar este artigo (Vancouver)}

Bernardes HC, Santos AFF, Gohm D, Dias LF, Vilges KMA, Oliveira SV. Análise epidemiológica dos casos de Leishmaniose Tegumentar Americana em um município do Triângulo Mineiro. REFACS [Internet]. 2020 [citado em inserir dia, mês e ano de acesso]; 8(1):67-77. Disponível em: inserir link de acesso. DOI: inserir link do DOI.

\section{Como citar este artigo (ABNT)}

BERNARDES, H. C.; SANTOS, A. F. F.; GOHM, D.; DIAS, L. F.; VILGES, K. M. A.; OLIVEIRA, S. V. Análise epidemiológica dos casos de Leishmaniose Tegumentar Americana em um município do Triângulo Mineiro. REFACS, Uberaba, MG, v. 8, n. 1, p. 67-77, 2020. Disponível em: inserir link de acesso. Acesso em: inserir dia, mês e ano de acesso. DOI: inserir link do DOI.

\section{Como citar este artigo (APA)}

Bernardes, H.C., Santos, A.F.F., Gohm, D., Dias, L.F., Vilges, K.M.A. \& Oliveira, S.V. (2020). Análise epidemiológica dos casos de Leishmaniose Tegumentar Americana em um município do Triângulo Mineiro. REFACS, 8(1), 67-77. Recuperado em: inserir dia, mês e ano de acesso de inserir link de acesso. DOI: inserir link do DOI. 